A N N A L E S Annales de Bretagne et des Pays de l'Ouest

Anjou. Maine. Poitou-Charente. Touraine

123-2 | 2016

Varia

\title{
Tant d'or que d'argent.
}

La monnaie en Basse-Normandie à l'époque moderne ( $\mathrm{XVI}^{\mathrm{e}}$-XvIII ${ }^{\mathrm{e}}$ siècle)

Yves Coativy

\section{OpenEdition}

1 Journals

\section{Édition électronique}

URL : http://journals.openedition.org/abpo/3346

DOI : $10.4000 / a b p o .3346$

ISBN : 978-2-7535-5185-5

ISSN : $2108-6443$

\section{Éditeur}

Presses universitaires de Rennes

\section{Édition imprimée}

Date de publication : 28 juillet 2016

Pagination : 194-197

ISBN : 978-2-7535-5183-1

ISSN : 0399-0826

\section{Référence électronique}

Yves Coativy, «Tant d'or que d'argent. », Annales de Bretagne et des Pays de l'Ouest [En ligne], 123-2 I

2016, mis en ligne le 28 juin 2016, consulté le 23 septembre 2020. URL : http://

journals.openedition.org/abpo/3346; DOI : https://doi.org/10.4000/abpo.3346 
leurs serviteurs de Châteaubriant. Dans le cas du Grand Condé par exemple, les dépouillements de l'auteur font découvrir au lecteur une figure moins connue que le héros de guerre, celle du grand seigneur, soucieux de la bonne gestion de ses affaires foncières. Et la baronnie de Châteaubriant ne devait être qu'une goutte d'eau dans un océan de possessions. Aussi, c'est toute la gestion seigneuriale d'immenses fortunes qui se trouve ici interrogée.

Il faut cependant constater l'absence de notes de bas de page qui ne permet pas de connaître les sources utilisées par l'auteur et cela est bien dommage lors de citations par exemple. Le lecteur n'en continue pas moins d'avoir confiance en l'auteur et l'on explique cela par des contraintes éditoriales à regretter. Néanmoins, ce défaut d'édition est compensé par la publication en fin de chapitres de documents originaux comme des extraits de procès, des lettres originales parfois issues des archives de Chantilly, des mémoires seigneuriaux, des listes d'aveux etc. dont les références sont cette fois-ci données. Ces documents font réellement vivre l'ouvrage et entraînent le lecteur dans la réalité sociale de la baronnie de Châteaubriant.

Ainsi faut-il saluer la publication de cette thèse, attendue depuis quelques années déjà. Elle constitue une avancée indéniable pour l'histoire de Châteaubriant et prouve que les grands fiefs d'Ancien Régime sont des mannes archivistiques. L'ouvrage montre que l'on peut faire apparaître des tensions sociales à l'échelle féodale. Surtout, il interroge le lecteur sur la gestion d'imposantes seigneuries par une très grande noblesse de cour pour qui les revenus fonciers restent des préoccupations majeures. On termine cette lecture avec l'impression, la conviction presque, que les liens féodaux entre grand seigneur et serviteurs sont parfois plus intéressants pour ces derniers que le service du roi.

Antoine RIVAuLT

JAMBU, Jérôme, Tant d'or que d'argent. La monnaie en Basse-Normandie à l'époque moderne (XVI'-XVIII siècle), Rennes, PUR, coll. " Histoire", 2013, 640 p., ill.

La monnaie médiévale est assez bien connue pour la France, même si du côté du monde féodal, il reste des zones d'ombre et de grandes friches. Il en va différemment pour l'époque moderne. Aux travaux importants qui ont marqué les années 1970 a succédé une période de désaffection pour le sujet qui s'achève avec l'ouvrage de Jérôme Jambu. Maître de conférences en histoire moderne à l'université de Lille 3-Charles de Gaulle, il a consacré sa thèse à la monnaie en Basse-Normandie et nous livre un ouvrage sur les ateliers de Saint-Lô et de Caen du XVI ${ }^{\mathrm{e}}$ au XvIII ${ }^{\mathrm{e}}$ siècle, fruit de sa thèse de doctorat conduite sous la direction de Jean-Marc Moriceau et soutenue à Caen en 2008. L'auteur a traité la question de façon thématique, de la fabrication des monnaies d'or et d'argent en atelier à leur usage par les populations. De très nombreuses cartes mais aussi des croquis et des illustrations viennent éclairer ce fort volume de 640 pages.

L'ouvrage s'organise en deux parties, la première traite de la production monétaire, la seconde de l'utilisation des espèces. La monnaie est au temps du trimétallisme, or, argent, bronze, à la fois un instrument des échanges, une réserve de valeur et un moyen pour le pouvoir de véhiculer un message politique auprès du plus grand nombre. J. Jambu commence son étude par l'atelier de Saint-Lô. Il est tenu au XVI ${ }^{\mathrm{e}}$ siècle par des locaux puis par des horsains qui viennent faire fortune, appuyés sur un réseau, malgré des tentatives de reprise en main par des Saint-Lois. Par contre, officiers et main-d'œuvre sont issus de la région, protégés par leurs 
privilèges, ils s'opposent à toute intrusion. Le rythme de la production est irrégulier, voyant alterner des périodes de calme et d'autres d'intense activité. Trois éléments peuvent être mis en avant pour expliquer ces variations : la politique royale, bien sûr, qui pousse ou non les contemporains à apporter leur métal aux Monnaies; la conjoncture économique et monétaire, mais aussi la capacité des maîtres particuliers à mobiliser du métal précieux. Quelques poussées majeures de production sont à souligner, comme dans les années 1550-1555 avec la refonte des billons qui fait affluer de grandes quantités de monnaies décriées et relance la production. À l'inverse, le règne de Louis XIII est marqué par une longue période de faible activité. Ensuite, Saint-Lô, malgré la résistance de son personnel, plie devant la concurrence de Caen et doit lui céder la place. En effet si le premier atelier relève du fonctionnement normal de l'administration, celui de Caen est créé pour favoriser les manipulations monétaires caractéristiques du règne de Louis XIV. Il supplante rapidement Saint-Lô et concentre la production de Basse-Normandie jusqu'à la fin du règne de Louis XV. La faiblesse de la production de Saint-Lô à la fin de la période entraine sa mise en sommeil.

L'atelier connaît pourtant au XVIII ${ }^{\mathrm{e}}$ siècle des heures fastes grâce aux arrivages réguliers de métal en provenance des Amériques. J. Jambu montre bien, preuves à l'appui, que l'or du Guatemala joue un rôle essentiel dans la production, à Saint-Lô comme à Caen. On notera le recours de l'auteur aux analyses chimiques non-destructives qui permettent d'apporter des éléments nouveaux sur les origines du métal. Il en va de même pour l'argent américain qui arrive sous forme de piastres ou de lingots en quantité non-négligeable, comme cette tonne et demie de piastres monnayée à Caen en 1709 et les presque cinq tonnes en lingots transformées en monnaie en $1711 \ldots$

L'étude des ateliers et de leurs conditions de production montre que le personnel de Saint-Lô est pléthorique, alors que celui de Caen est en nombre raisonnable au vu de la taille de l'atelier. Comme ailleurs, ils se divisent entre personnel de direction (directeur, garde, avocat, graveur, essayeur...) et monnayeurs et ajusteurs chargés de la frappe.

J. Jambu se penche dans sa seconde partie sur l'utilisation quotidienne de la monnaie, en croisant les sources. Cela le conduit à remettre en cause un topos de l'histoire moderne qui veut que l'économie des campagnes soit faiblement monétisée. Les populations voient passer des monnaies, de billon mais aussi d'or et d'argent, et l'impôt, au moins depuis le bas Moyen Âge, oblige les familles à stocker un peu de métal précieux en vue des prélèvements obligatoires. D'autre part, la pénurie de monnaie est rare. Elle concerne surtout les petites pièces peu rentables à fabriquer. Face à cette situation, les populations s'adaptent en ayant recours au troc notamment. Mais l'auteur montre bien que l'on a beaucoup exagéré la disette monétaire. Par ailleurs, pour les sommes plus importantes, le papier sert efficacement à suppléer la monnaie.

Des professionnels sont présents pour animer la circulation monétaire, aider à stocker le métal et à le redistribuer. Les ateliers monétaires jouent, bien sûr efficacement, ce rôle, de même que les changeurs. Ces derniers assurent le prélèvement des fausses pièces, le change des monnaies étrangères, anciennes ou décriées. Les listes dressées par J. Jambu indiquent leur présence dans les grandes villes mais aussi dans des endroits moins actifs du point de vue économique comme Domfront, Falaise, Vire, etc. Les trésoreries de l'État et les banquiers font le reste et tout le monde vit au rythme des foires de Caen et de Guibray qui permettent, entre autres choses, de liquider les billets à terme, de clôturer les comptes et d'alimenter le commerce en monnaies de toutes origines. Se pose aussi pour les populations le problème de conserver et de transporter de l'argent, ce qui ne va pas sans difficulté. 
Les uns enterrent leurs économies, d'autres les confient à un coffre-fort, mais les mesures de protection sont plus drastiques quand il s'agit, comme le 8 août 1716 , de transporter plus de 3 tonnes d'argent de Caen à Paris!

La circulation monétaire générale est ensuite abordée en deux chapitres. J. Jambu distingue trois périodes, celle de l'or, jusque vers 1550, celle de l'argent jusque vers 1640 et celle du billon, alors que l'or revient en force à la fin de la période. Il montre aussi qu'au-delà de la théorie, il existe des usages curieux (et anciens) qui consistent à utiliser des monnaies décriées ou à surévaluer certaines espèces sans tenir compte de leur cours officiel. En 1538, par exemple, le royal d'or dont le cours officiel est de 44 sous 6 deniers vaut au cours réel 50 sous, preuve de la confiance que les populations ont en cette monnaie. Une réforme adoptée en 1577 met fin à ces pratiques, même si les campagnes gardent pendant quelque temps leurs mauvaises habitudes. J. Jambu aborde ensuite l'invention des louis d'or et d'argent et les conséquences que ces nouvelles espèces ont sur l'économie normande. L'effet principal est de faire disparaître en partie les monnaies anciennes et étrangères, mais les manipulations monétaires de l'époque de Louis XIV viennent perturber le fonctionnement du nouveau système et aggravent la " disette des espèces ". Le retour à la stabilité en 1726 stabilise la circulation monétaire pour longtemps.

Les monnaies sont concurrencées ou complétées par des billets de toutes sortes. Créés en 1701, les "billets de monnoye " circulent pendant une dizaine d'années mais souffrent de la méfiance des usagers qui préfèrent le métal au papier. Une deuxième tentative ne rencontre pas un franc succès avec les billets d'État (1715-1719). C'est surtout l'expérience de Law (1719-1720) qui enterre pour longtemps la monnaie fiduciaire. L'absence de confiance dans le papier-monnaie explique ces échecs répétés mais aussi le fait que les billets de monnaie n'étaient pas fractionnables : les billets d'État sont un moyen de paiement plus qu'une monnaie à proprement parler.

L'ouvrage se termine sur le faux-monnayage et sa répression. On sait que l'imagination des fraudeurs est sans limite, mais trois pratiques dominent : le rognage, la détention d'espèces décriées et la fabrication de monnaies fausses. Toutes ces activités sont passibles de peines très graves, ce qui n'empêche pas certains de s'y livrer, et même le clergé n'échappe pas à la tentation. J. Jambu raconte, par exemple, l'histoire de Louis de Renty, ancien abbé de Saint-Sever, qui est arrêté en juillet 1618 avec en sa possession des coins pour frapper de la fausse monnaie. Il s'empresse de demander à être jugé par la justice ecclésiastique, réputée plus souple. Grâce à un dossier très complet, J. Jambu reconstitue également une affaire de faux-monnayage, de sa découverte à sa sanction, qui, dans les années 1720-1730, se développe dans un rayon de dix kilomètres autour de Périers. En Normandie comme ailleurs, les peines très lourdes prévues par la justice sont appliquées : deux faux-monnayeurs sont bouillis à Caen en 1508, d'autres sont pendus dans la première moitié du XVII ${ }^{\mathrm{e}}$ siècle, tandis que le bannissement, les galères, les fustigations et les mutilations ne sont pas rares. Au-delà de l'aspect spectaculaire, une partie du chapitre est consacrée à la quantification du phénomène à partir des grandes fontes ordonnées dans les années 1720-1730.

L'ouvrage comporte encore des " Annexes " (125 pages), sept cartes, une chronologie, un lexique, l'indication des sources et de la bibliographie, ainsi qu'un index. Les sources utilisées et détaillées en fin de volume sont particulièrement nombreuses et prouvent que l'auteur a été chercher l'information de façon très fine. De même, la bibliographie, même allégée pour la publication, est particulièrement fournie et permettra à tous ceux qui s'intéressent à l'histoire économique de trouver leur bonheur.

Les critiques sont vénielles. On relèvera tout d'abord les citations d'autorités en tête de chapitre qui ont un côté très désuet. Bien plus problématique est le choix de 
différencier les ateliers monétaires de Saint-Lô et de Caen, ce qui hache le récit et a pour résultat des redites. Il aurait sans doute été préférable de traiter l'ensemble de façon synthétique, ce qui aurait permis de réduire le nombre de pages, au moins pour certains chapitres. L'étude d'ateliers monétaires dans les provinces à l'époque moderne ouvre une voie nouvelle à la recherche. Elle appelle d'autres travaux du même genre qui permettront des comparaisons.

Yves CoATIVY

ButI, Gilbert, CABANTous, Alain, Etre marin en Europe occidentale, 1550-1850, Rennes, PUR, coll. " Didact Histoire ", 2016, 225 p.

Conformément aux règles de cette collection, les deux auteurs entraînent le lecteur dans l'atelier de l'historien pour lui faire découvrir une série de vies de marins ou de générations de marins français d'une même famille et présenter la diversité des trajectoires. Ce sont des fratries de pêcheurs de génération en génération, des marins qui alternent pêche et commerce, des pêcheurs-paysans, une famille en difficulté quand le père et son fils disparaissent dans un naufrage, une famille qui accomplit sur plusieurs générations une reconversion du long cours vers l'ostréiculture... Ce sont aussi des profils d'ascension professionnelle et sociale : le marin devient propriétaire d'un domaine viticole, des marins d'estuaires accèdent au négoce et à l'armement, des fils de familles deviennent officiers de la Marine. Les auteurs nous plongent ensuite au cœur d'un ensemble documentaire, avec un florilège de textes et tableaux pris dans d'autres pays maritimes, et un superbe cahier iconographique de 32 pages illustrant les questions du recrutement, de la formation, du travail et de la vie à bord, du costume, du risque, de la mort et de Dieu.

Être marin se décline en plusieurs volets et plusieurs questions. C'est d'abord une profession qui s'apprend à bord dans un environnement familial ou de voisinage, seuls les maîtres et capitaines accédant à une formation dans les écoles d'hydrographie. L'auto-renouvellement, particulièrement fort dans les pêches, mais moindre dans le commerce maritime, ne peut empêcher le recours à des jeunes issus des métiers de l'agriculture, de l'artisanat et du commerce. Quant au recrutement du grand corps de la Marine, il s'est progressivement replié sur les jeunes nobles qui accèdent aux écoles des gardes. L'exercice du métier de marin se fait sous le contrôle de l'État. Pour les armements à la pêche et au commerce, celuici se décharge de la formation des équipages de la Marine qui sont soumis à la conscription obligatoire du système des classes puis de l'inscription maritime, système contesté parfois, finalement accepté grâce aux mesures d'accompagnement. Mais ces marins pratiquent des navigations plurielles, à bord de flottes très typées, au sein d'équipages aux effectifs très variables, et à la composition et la hiérarchie bien différentes, tout comme le sont les rémunérations à la solde ou à la part. Même si l'ancrage local dans la pêche et le cabotage lui donne une plus grande cohésion, l'équipage est une communauté éphémère.

Comment se comporte(nt)-t-il(s) en mer? Le navire est un huis clos où règnent la promiscuité, la crainte de voir les vivres détériorées, une hiérarchie et un ordre strict sous la direction du capitaine, l'exercice par quart de tâches multiples, un langage particulier. Malgré le souci croissant de l'hygiène, les hardes laissent peu de place au rechange et la panoplie du travailleur de la mer ne se met en place que progressivement. Ils sont tous soumis aux risques des maladies, des accidents, et l'usure par le métier fait vieillir plus vite. Le corps ainsi marqué est aussi un moyen 